\title{
The Evaluation of Refrigerated and Frozen Osteochondral Allografts in the Knee
}

\author{
Albert Washington Pearsall IV ${ }^{1}$, Sudhakar Govindarajulo Madanagopal ${ }^{1}$, Joseph Allan Tucker ${ }^{2}$ \\ ${ }^{1}$ Department of Orthopaedic Surgery, College of Medicine, University of South Alabama, Mobile, US \\ ${ }^{2}$ Department of Pathology, College of Medicine, University of South Alabama, Mobile, US \\ E-mail: apearsal@usouthal.edu \\ Received May 6, 2011; revised June 27, 2011; accepted July 10, 2011
}

\begin{abstract}
Between 1998 and 2002, 25 patients who were treated with a refrigerated or frozen allograft were evaluated. The mean patient age was 48 years. The mean lesion size was $4.5 \mathrm{~cm}^{2}$. Validated outcome instruments [Knee Society Score, Western Ontario and McMaster University Score] were used. Clinical and radiographic evaluations were performed pre-operatively and at the most recent follow-up. Histological and electron microscopic analysis was performed on grafts prior to implantation. Clinical follow-up averaged 46 months (range 24 - 60 months). The Western Ontario and McMaster University Score improved from $46+24$ to $66+22$ (p $=0.003)$. The Knee Society Score improved from $104+43$ to $132+42(\mathrm{p}=0.01)$. No correlation was noted between graft type and histological or electron microscopy scoring. Post-operative mechanical alignment was not correlated with an improvement in Western Ontario and McMaster University Score $(\mathrm{p}=0.19)$ or Knee Society Score (0.27). Six patients (24\%), all refrigerated allografts, were failures and underwent knee arthroplasty. Seventy-six percent of implanted frozen and refrigerated osteochondral allografts are in place 4 years after surgery. Frozen allografts appear to be surviving as well as refrigerated grafts. The use of magnetic resonance imaging may enable the evaluation of graft incorporation and articular cartilage integrity.
\end{abstract}

Keywords: Allograft, Refrigerated, Frozen, Knee, Transplantation

\section{Introduction}

Biologic treatment options for large full thickness osteochondral lesions include microfracture, autologous cartilage transplantation, mosaicplasty and refrigerated or frozen osteochondral allograft transplantation [1-6]. The result of these treatments is partial defect filling with fibrocartilage consisting of predominately type I collagen. Fibrocartilage has diminished resilience and a predilection for deterioration over time [2,3,5,7-10].

The use of fresh osteochondral allografts in the treatment of full thickness articular cartilage defects has been well documented, with success rates of $75 \%$ reported at 5 years, slightly deteriorating to $63 \%$ at 14 years [8,11-18]. The term "fresh" usually indicates graft harvest within 24 hours of the donor's death and a time from graft harvest to implantation of 7 days or less $[2,9,10,12,13$, 19-24]. Deep frozen allografts have also been used for reconstruction of osteoarticular defects. However, authors have cited the diminished cell viability and potential matrix degeneration that can occur after freezing hyaline cartilage [11,25-28].

Previously, authors have published the viability results of stored refrigerated allografts $[3,29,30]$. However, the authors are unaware of data correlating patients' functional outcome with radiographic evaluation and histological/ electron microscopy grading of refrigerated and frozen allografts at the time of implantation. The purpose of the current study was to clinically and radiographically evaluate patients who underwent refrigerated or frozen allograft transplantation. In addition, histological/electron microscopy grading of the allograft and radiographic evaluation of the affected knee was analyzed in relationship to functional outcome at an average of 46 months follow-up.

\section{Materials and Methods}

\subsection{Patient Data}

The current study was approved by the Institutional Review Board at our institution prior to implementation. All 
study patients gave informed consent prior to being enrolled in the study. Between 1998 and 2002, 26 patients underwent osteoarticular transplantation of the femur and/or patella with a refrigerated or frozen allograft from which histologic and electron microscopic data was available at the time of implantation. Inclusion criteria were as follows: 1) Tegner 3 or greater activity level; and 2) a contained articular cartilage defect amenable to a non-structural osteoarticular graft; 3) articular cartilage damage limited to 1 or 2 compartments; 4) biomechanical knee alignment that was less than $5^{\circ}$ of varus or valgus or correctable with a distal femoral or proximal tibial osteotomy; and 5) failure of conservative measures including non-steroidal anti-inflammatory medications and physical therapy for a minimum of 3 months. Body mass index greater than 30 and chronological age were not used as exclusionary criteria from surgery. Patients whose lifestyle included less demanding activities were encouraged to undergo a unicondylar or total knee arthroplasty.

Prior to surgery, all patients underwent clinical evaluation, standing radiographic imaging of both knees, and magnetic resonance imaging of the affected knee. Specific magnetic resonance imaging sequences (fast spin echo) were performed to assess the articular cartilage of the patella, femur and tibia. All images were reviewed by the senior author and a board certified, fellowshiptrained radiologist.

\subsection{Operative Technique}

All allograft transplants were performed through a mini-arthrotomy unless a tibial tubercle osteotomy was performed for a patellar and/or trochlear defect. After the recipient defect was measured, the bone was reamed to a depth of 8 and $12 \mathrm{~mm}$. The donor allograft was cored with a coring device (Arthrex, Inc., Naples, Florida) with a minimum diameter of $18 \mathrm{~mm}$. The graft was press-fit into the recipient site with the articular surfaces congruent. All grafts were placed with less than $1 \mathrm{~mm}$ of donor-recipient articular surface incongruity. Early in the study, 1 graft (patella) was secured with an intra-articular screw. No subsequent grafts underwent fixation.

All tibial tubercle osteotomies were performed according to the technique described by Fulkerson and secured with two $3.5 \mathrm{~mm}$ or $4.5 \mathrm{~mm}$ screws [24] .All high tibial osteotomies were performed with a lateral closing or medial opening wedge. All distal femoral osteotomies were performed with a lateral opening wedge. For all opening wedge osteotomies, frozen allograft corticocancellous bone graft was used to fill the osteotomy defect.

\subsection{Postoperative Treatment}

All patients undergoing an isolated mini-arthrotomy were discharged the following day. Patients undergoing a tibial tubercle osteotomy, high tibial osteotomy or distal femoral osteotomy remained in the hospital 48 to 72 hours. For all inpatients, continuous passive motion was instituted on the first post-operative day. Continuous passive motion was continued on an outpatient basis 3 to 6 hours per day for 3 weeks post-operatively. Patients undergoing isolated allograft transplantation were kept toe-touch weight bearing for 4 weeks and then progressed to full weight bearing over 2 weeks. All patients undergoing a tibial tubercle osteotomy, high tibial osteotomy or distal femoral osteotomy were kept non-weight bearing for 6 weeks and progressed to full weight- bearing over the next 2 weeks. Heavy labor and athletic activity were delayed until 6 months after surgery.

\subsection{Clinical and Radiographic Assessment}

All patients underwent radiographic evaluation preoperatively and at yearly intervals. Two standardized radiographic views (standing antero-posterior and skyline) were obtained by a trained radiological technician. In addition, standing long-leg antero-posterior radiographs were obtained pre-operatively and at the most recent follow-up. A trained research assistant blinded to the patient's clinical data measured each film. Each film was assessed for the area of greatest joint space narrowing (Grade $3=>3 \mathrm{~mm}$ joint space, Grade $2=<3 \mathrm{~mm}$ of joint space but not bone on bone, Grade $1=$ bone on bone) in specific areas (Figure 1). Based upon these measurements, the patient was given a composite score ranging from 3 to 6 which was used for later analysis. Long leg weight-bearing radiographs were measured by one of the authors. The biomechanical axis was determined by the intersection of a line drawn from the center of the femoral head to the center of the tibial plateau and a line drawn from the center of the talus to the center of the tibial plateau.

All patients underwent clinical evaluation including knee range of motion. Patients completed the Knee Society Score, Western Ontario and McMaster University (WOMAC) Score preoperatively and at yearly intervals after surgery. Failure was defined as conversion of a transplanted graft to a unicondylar or total knee arthroplasty. All failures were included in the overall analysis and also analyzed separately.

\subsection{Refrigerated Allografts}

All grafts were aseptically processed within 72 hours of 


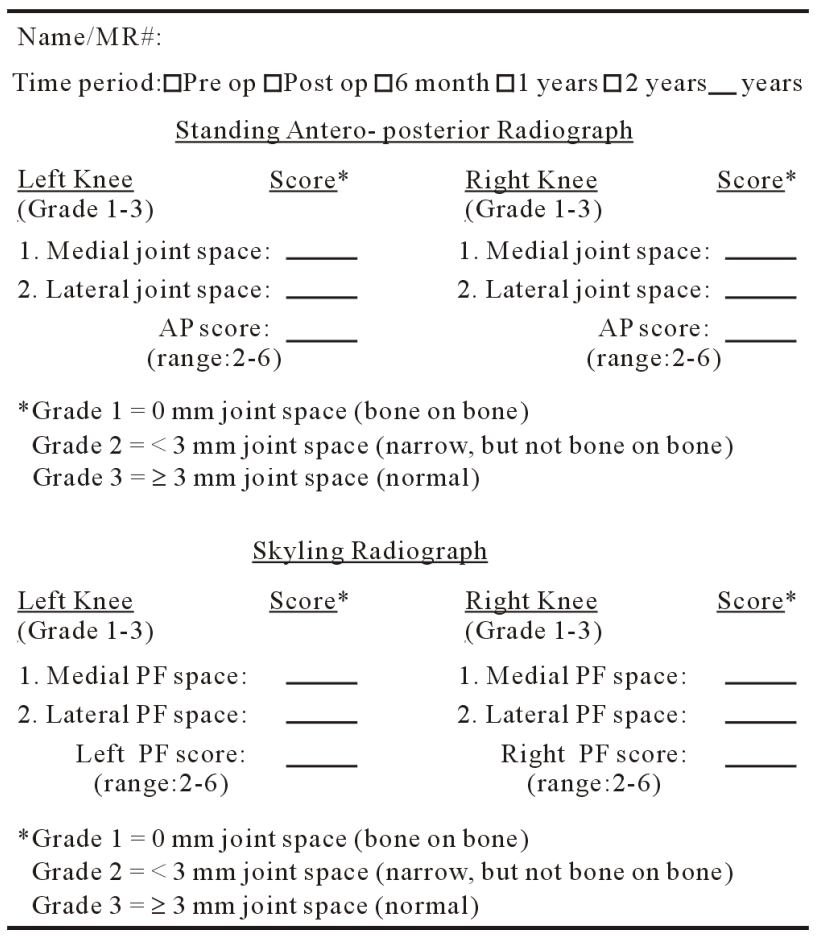

Figure 1. Knee radiographic grading form. A grade from one to three is given for the medial and lateral joint spaces within the knee and patellofemoral joint.

the donor's death and procedures were performed in accordance with the guidelines established by the American Association of Tissue Banks. Donors' blood was screened for malignancies, autoimmune and certain neurological disorders, and any high-risk behavior. Donor blood and tissue was screened for human immunodeficiency virus-1 and human immunodeficiency virus-2 antibodies, Hepatitis B and C antibodies, human T-lymphotropic Virus I and II, syphilis, and polymerase chain reaction testing for human immunodeficiency virus-1.

Harvested osteochondral grafts were aseptically cleansed with saline pulse lavage in order to remove blood and fat from the cancellous bone. All grafts were pre-soaked in antibiotic storage medium. Grafts were transferred to double sterile pouches containing storage media to maximize chondrocyte viability during storage (INCELL Corp., San Antonio, Texas). Only grafts demonstrating no growth after 7 day microbial culturing were released for distribution. Grafts were refrigerated and stored at $2^{\circ}-8^{\circ}$ Celsius with a shelf life of 6 weeks from the date of processing (Regeneration Technologies Inc., Alachua, Florida).

\subsection{Frozen Allografts}

All grafts were aseptically processed within 72 hours of the donor's death and procedures were performed in ac- cordance with the guidelines established by the American Association of Tissue Banks. Donors' blood was screened for malignancies, autoimmune and certain neurological disorders, and any high-risk behavior. Donor blood and tissue was screened for human immunodeficiency virus-1 and human immunodeficiency virus-2 antibodies, Hepatitis B and C antibodies, human Tlymphotropic Virus I and II, syphilis, and polymerase chain reaction testing for human immunodeficiency virus-1. All grafts were stored at $-70^{\circ}$ Fahrenheit until the day of use.

\subsection{Cartilage Preparation and Histologic Scoring}

Immediately upon opening the allograft in the operating room, three $5 \mathrm{~mm}$ plugs were sterilely harvested from a peripheral area of articular cartilage with an Osteochondral Autograft Transfer System (OATS) harvester (Arthrex, Naples, Florida). The sample site was free from any visual cartilage defects. All plugs were placed in a sterile container with a small amount of normal saline and sealed for transport to the pathology and flow cytometry laboratories for evaluation. The time from plug harvest to processing was approximately 30 minutes for all specimens.

For histology a portion of fresh cartilage was cut from the bone surface, fixed in $10 \%$ buffered formalin, processed to paraffin, sectioned at $5 \mathrm{um}$, and stained with hematoxylin and eosin. Three slides were prepared from the plugs and sent for evaluation. The slides were scored blindly and the scores averaged. The average score was used for data analysis. The following scoring system was utilized for each slide: 0: all cells appeared lethally injured; 1+: majority of cells exhibited marked to lethal injury; 2+: minority of cells exhibited marked to lethal injury; $3+$ : all cells appear viable. Severe pyknosis and cell lysis were judged to represent marked to lethal injury.

For electron microscopy, fresh tissue was placed in $3 \%$ glutaraldehyde $(0.1 \mathrm{~N}$ sodium cacodylate buffer, $\mathrm{pH}$ 7.2 ), post-fixed in $1 \%$ osmium tetroxide, and embedded in epoxy resin. Sections (1 um) were stained with toluidine blue. Thin sections were stained with uranyl acetate and lead citrate and examined in a Phillips CM100 electron microscope (FEI Company, Hillsboro, Oregon). The scoring scheme above was also applied to the ultrastructural findings. Features such as severe pyknosis, obscured detail of the organelles, and rupture of the plasma membrane were considered indicators of lethal injury, and lesser degrees of cytoplasmic contraction, cytoplasmic blebs, and accumulation of myelin figures were considered indications of marked injury (Figures 2(a)-2(c)). 


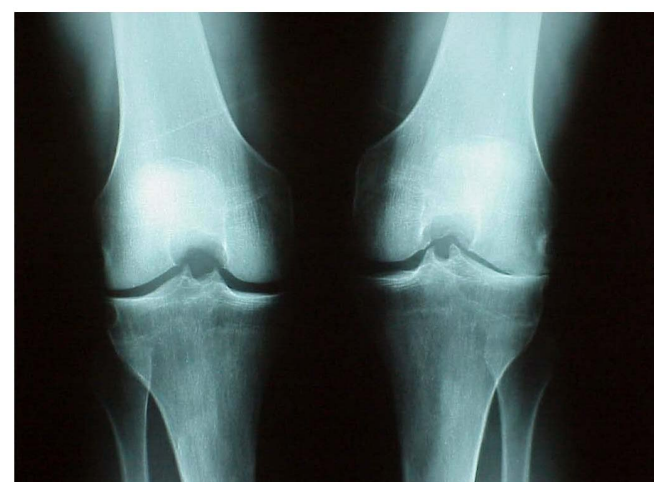

(a)

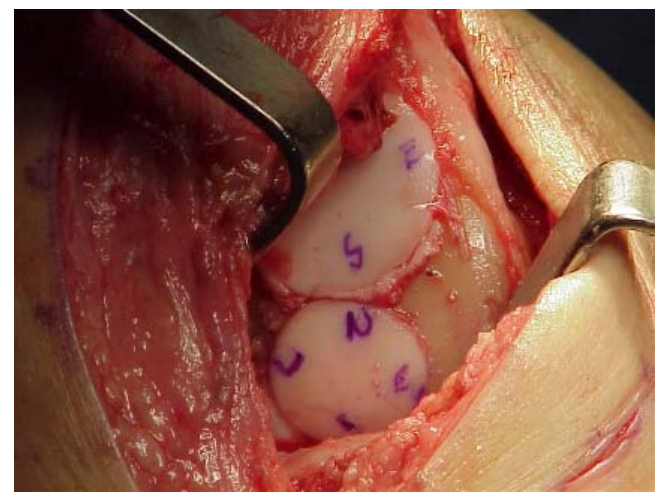

(b)

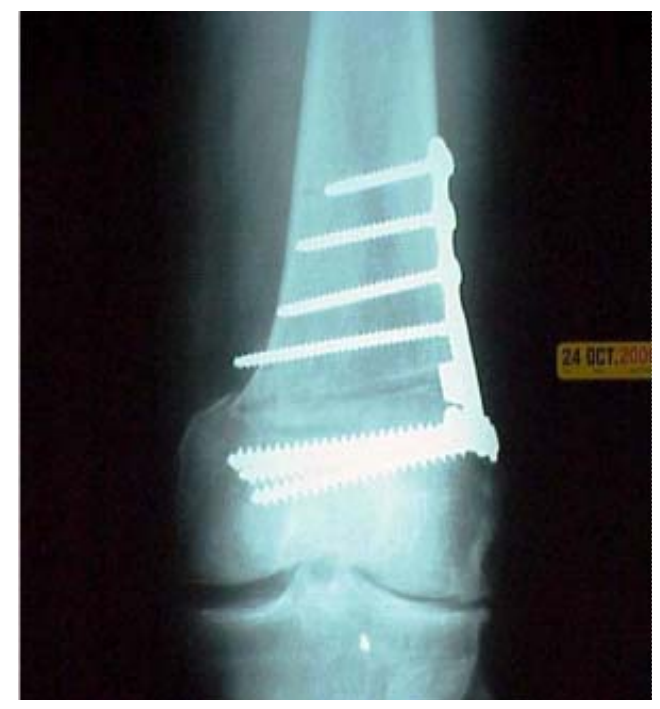

(c)

Figure 2. (a)-(c) Pre-operative and post-operative radiographs and intraoperative radiographs and intra-operative images of an active male farmer who underwent a distal femoral opening wedge osteotomy and lateral femoral condyle refrigerated osteoarticular allograft. (a) Pre-operative standing antero-posterior radiograph. The patient's preoperative KSS score was 175. (b) Intra-operative image from the previous patient showing two refrigerated osteoarticular plugs in place in the lateral femoral condyle. (c) Post-operative standing antero-posterior radiograph of the same patient. His post-operative KSS score at the latest follow-up was 190.

\subsection{Statistical Analysis}

Statistical analyses were performed using appropriate procedures of the JMP software system (SAS Inc., Carey, NC). Means for demographics, graft size, follow-up, shelf time (time from harvest to implantation), harvest time (time of death to time of harvest), electron microscopy score and histology score were calculated. Correlation analysis were performed to determine if a relationship existed between electron microscopy score, histology score, outcome score and any other variable. Significance was determined at the 0.05 level.

\section{Results}

Twenty-five patients (96\%) with complete data were available for follow-up. The average age for the overall group was 48 years. The average follow-up for the group was 46 months. The average body mass index of the group was 32. The average graft size for the group was $4.5 \mathrm{~cm}^{2}$. Pre-operative knee range of motion was less in the frozen group when compared to the refrigerated group ( $\mathrm{p}=0.01)$ (Table 1).

Both groups improved after surgery. Post-operative WOMAC Score, Knee Society Score and knee range of motion values were 66, 132, and 116 respectively (Table 2) (Figures 2(a)-2(c)). Post-operative improvement in WOMAC Scores and Knee Society Scores, and knee range of motion was analyzed in relation to graft type. Greater improvement in WOMAC and Knee Society Scores was noted in the frozen group when compared to the refrigerated group $(\mathrm{p}=0.07)$ (Figures 3(a)-3(b)). An improvement in knee range of motion was noted in frozen patients compared refrigerated allograft patients $(\mathrm{p}=$ 0.02) (Table 2).

Table 1. Demographic variables for refrigerated and frozen allograft patients.

\begin{tabular}{llll}
\hline Variable & Refrigerated & Frozen & p-value \\
\hline Age & $44(17-69)$ & $57(35-66)$ & $\mathbf{0 . 0 2} *$ \\
Sex (male/female) & $10 / 8$ & $1 / 8$ & $\mathbf{0 . 0 5} *$ \\
BMI & $30(20-48)$ & $35(25-43)$ & 0.11 \\
Follow-up (months) & $47(24-60)$ & $44(35-57)$ & 0.47 \\
Number of plugs & $2.6(1-5)$ & $2.2(1-4)$ & 0.53 \\
Size (cm $)$ & $4.2(2.5-7.3)$ & $5.3(2.0-8.5)$ & 0.20 \\
Pre-op pain & $3 / 4(1-4)$ & $4 / 4(1-4)$ & 0.15 \\
Pre-op WOMAC & $52(6-94)$ & $35(19-63)$ & 0.1 \\
Pre-op KSS & $113(30-192)$ & $85(30-130)$ & 0.12 \\
Pre-op ROM & $118(95-135)$ & $99(50-130)$ & $\mathbf{0 . 0 1 *}$ \\
\hline
\end{tabular}

*Statistically significant. 
Table 2. Overall pre and post-operative outcome scores and outcome improvement in refrigerated and frozen allograft patients.

\begin{tabular}{cccc}
\hline $\begin{array}{c}\text { Outcome } \\
\text { Measure }\end{array}$ & $\begin{array}{c}\text { Pre-operative } \\
\text { score }\end{array}$ & $\begin{array}{c}\text { Post-operative } \\
\text { score }\end{array}$ & P value \\
\hline WOMAC & $46+/-24$ & $66+/-22$ & $0.003 *$ \\
KSS & $104+/-43$ & $132+/-42$ & $0.01^{*}$ \\
Range of motion & $112+/-19$ & $116+/-11$ & 0.22 \\
\hline $\begin{array}{c}\text { Outcome } \\
\text { Measure }\end{array}$ & Refrigerated & Frozen & P value \\
\hline $\begin{array}{c}\text { WOMAC } \\
\text { improvement } \\
\text { KSS }\end{array}$ & $10+/-21$ & $28+/-21$ & 0.07 \\
$\begin{array}{c}\text { improvement } \\
\text { ROM }\end{array}$ & $14+/-35$ & $49+/-52$ & 0.07 \\
improvement & $-3+/-15$ & $20+/-30$ & $0.02 *$ \\
\hline
\end{tabular}

*Statistically significant.

Radiographic measurements were made on standing antero-posterior (AP) and skyline views. Medial and lateral tibio-femoral joint spaces were given a score from 1 to 3 , while medial and lateral patello-femoral joint spaces were given a score from 1 to 3 . The baseline group AP radiographic score was 5.1 which increased at follow-up to $5.3(\mathrm{p}=0.06)$. The baseline patella score for the group was 5.6 which increased at follow-up to 5.7 (Table 3).

Mechanical axis was measured in all patients without a tibial tubercle osteotomy. The average pre-operative and post-operative mechanical axes were $174^{\circ}$ and $177^{\circ}$ respectively $(p=0.08)$. When post-operative alignment was analyzed in relation to outcome improvement, no correlation was noted (Table 4).

Due to the small numbers, the 4 histological and electron microscopy scores were combined (scores zero and one $=$ Grade $1 /$ scores two and three $=$ Grade 2). When pre-operative WOMAC and Knee Society Scores were compared, there was no difference between groups. No correlation was noted between graft type and histological or electron microscopy scoring. Therefore, no difference in chondrocyte viability was noted on histological or electron microscopy between refrigerated and frozen allografts at the time of implantation. No correlation was noted between the histological or electron microscopy grading systems and any outcome tool (Table 5).

Improvement in outcome was analyzed in relation to the number of sites undergoing grafting. No difference in outcome improvement was noted between single or multiple site grafts (Knee Society Score $\mathrm{p}=0.7$; WOMAC Score $\mathrm{p}=0.5$; range of motion $\mathrm{p}=0.9$ ).

No difference in post-operative scores was noted between the 2 groups $(p=0.8, p=0.7)$. However, poorer

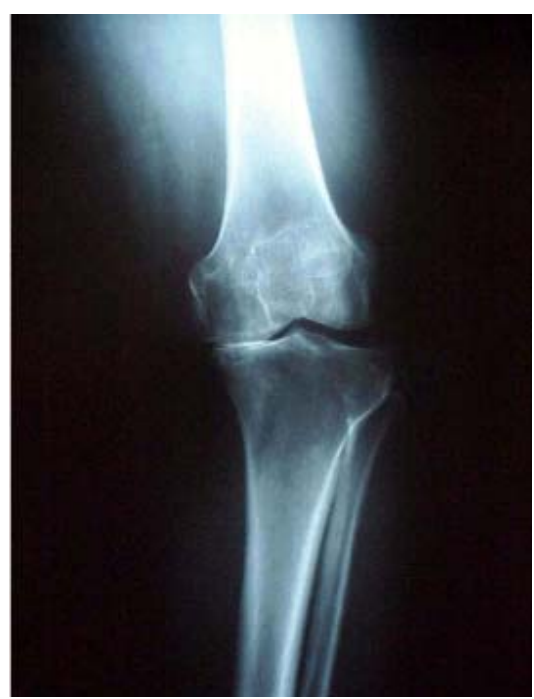

(a)

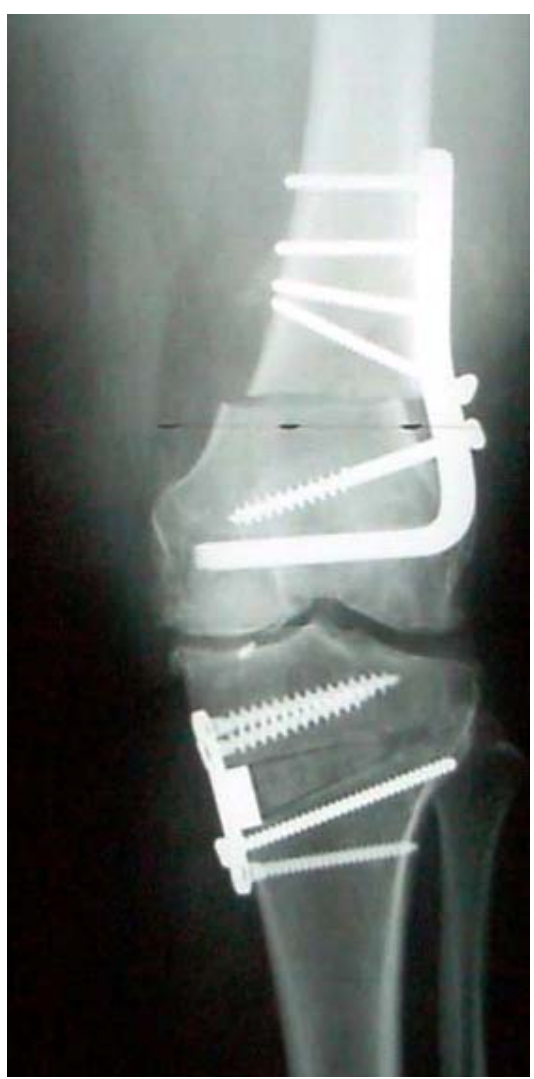

(b)

Figure 3. (a), (b) Pre-operative and post-operative radiographs of a homemaker with 22 degrees of combined femoral and tibial varus. She underwent combined distal femoral closing wedge and medial tibial opening osteotomies. A frozen osteoarticular allograft was used to reconstruct the medial femoral condyle. (a). Pre-operative stan- ding antero-posterior radiograph. The patient's pre-ope- rative KSS score was 59. (b). Post-operative standing antero-posterior radiograph of the same patient. Her post-ope- rative KSS score at the latest follow-up was 185. 
Table 3. Pre-operative and post-operative radiological scores for all patients $(n=25)$.

\begin{tabular}{llll}
\hline Score & Pre-operative & Post-operative & P value \\
\hline AP Score & $5.1+/-0.96$ & $5.3+/-1.18$ & 0.6 \\
Patella Score & $5.6+/-1.12$ & $5.7+/-1.36$ & 0.5 \\
\hline
\end{tabular}

Table 4. Analysis of post-operative mechanical alignment in relation to improvement in outcome score.

\begin{tabular}{ccccc}
\hline Outcome & $\begin{array}{c}\text { Varus } \\
(\mathbf{n}=\mathbf{1 2})\end{array}$ & $\begin{array}{c}\text { Neutral } \\
(\mathbf{n = 2})\end{array}$ & $\begin{array}{c}\text { Valgus } \\
(\mathbf{n}=3)\end{array}$ & P value \\
\hline WOMAC & $6+/-6$ & $34+/-15$ & $24+/-13$ & 0.19 \\
KSS & $31+/-12$ & $54+/-31$ & $-9+/-25$ & 0.27 \\
$\begin{array}{c}\text { Knee Range } \\
\text { of motion }\end{array}$ & $6.4+/-6.7$ & $22.5+/-17$ & $1.6+/-14$ & 0.33 \\
\hline
\end{tabular}

Table 5. Evaluation of histological and electron microscopy grades compared to outcome measures.

\begin{tabular}{cccc}
\hline \multicolumn{4}{c}{ Histology grading versus outcome measures } \\
\hline Outcome measures & Grade 1 & Grade 2 & P value \\
WOMAC improvement & 10.66 & 21.63 & 0.25 \\
KSS improvement & 24.41 & 24.81 & 0.9 \\
ROM improvement & -1.6 & 10.45 & 0.2 \\
\hline Electron microscopy grading versus outcome measures \\
\hline Outcome measures & Grade 1 & Grade 2 & P value \\
WOMAC improvement & 13.73 & 21.75 & 0.5 \\
KSS improvement & 23.93 & 41.25 & 0.5 \\
ROM improvement & 1.4 & 15.25 & 0.29 \\
\hline
\end{tabular}

pre-operative knee range of motion, Knee Society and WOMAC scores in patients receiving a frozen allograft $(p=0.01, p=0.1, p=0.1)$ resulted in a greater net change in outcome in the frozen allograft group.

Six patients (24\%) who underwent a knee arthroplasty were considered failures and analyzed separately. All failures were refrigerated allografts. We evaluated histological and electron microscopy findings at the time of implantation with success or failure of the graft. No failures were noted if the histology score was Grade $2(\mathrm{p}=$ $0.02)$ or if the electron microscopy score was Grade 2 ( $p$ $=0.4$ ).

\section{Discussion}

The use of fresh allografts for the treatment of full thickness articular defects is well documented $[2,15$, $31-34]$. Fresh osteochondral shell allografts $(<1 \mathrm{~cm}$ of subchondral bone) provide the greatest likelihood of chondrocyte survivability, while reducing immunogenicity by decreasing the exposure of white cells found in cancellous bone [1]. Various authors have indicated that the long-term survival of an allograft is dependent upon viable chondrocytes with a high level of donor deoxyribonucleic acid (DNA) to replenish the transplanted matrix [35]. Currently, fresh allografts provide the largest population of viable donor chondrocytes for transplantation.

Although fresh osteochondral allografts provide an excellent source of live chondrocytes, their use is not without drawbacks. Foremost is the need for the graft to be as fresh as possible to maintain chondrocyte viability, yet not expose the recipient to infection at the time of implantation. Currently, all published reports of osteochondral grafts using the term "fresh" have cited a time from graft harvest to implantation of 7 days or less [4,36-44]. In the current study, the time from graft harvest to implantation was greater than 7 days for all non-frozen grafts. Noting this, we chose to label these grafts as "refrigerated", rather than "fresh".

Recent data have indicated good results with the use of refrigerated osteochondral allografts. Emmerson et al. reported $72 \%$ good/excellent results at 7.7 years with refrigerated allografts averaging $7.5 \mathrm{~cm}$ in size implanted for osteochondritis dissecans of the femoral condyle. [45]. McCulloch et al. reviewed 25 consecutive patients who underwent refrigerated osteochondral allograft transplantation for defects in the femoral condyle. Overall, patients reported $84 \%$ satisfaction with their results [46]. Williams et al. reported on 19 patients who were treated with "fresh stored" allografts maintained for an average of 30 days prior to implantation. Mean lesion size was $602 \mathrm{~mm}^{2}$. At the most recent follow-up, the mean Short Form-36 score improved to $66(+/-24)$ and the mean score on the Activities of Daily Living Scale increased to $70(+/-22)$ [47].

The outcome tools used in the current study were not identical to those used by other authors $[46,47]$. However, both the WOMAC and Knee Society Scores are validated outcome instruments reported in the literature for the evaluation of patients with arthritic conditions [4850]. Indeed, previous studies of function in osteoarthritis have shown that WOMAC Score is more sensitive to change and has greater efficiency than other instruments used to assess osteoarthritis, including the SF-36 Health Survey [51,52].

In the current study we noted clinical improvement in patients implanted with a refrigerated or frozen allograft. Many of these patients had malalignment or patellofemoral disease requiring a concomitant osteotomy. Whe- ther the osteotomies themselves played a larger 
role then the transplantation in improved patient outcome is unclear.

Numerous animal studies have indicated that harvested osteochondral allografts can be safely preserved up to 28 days with overall maintenance of biomechanical properties, cell matrix collagen content, and permeability [53-56]. Wayne et al. demonstrated that osteochondral shell grafts could be stored in culture medium at $4^{\circ} \mathrm{Cel}-$ sius for up to 60 days without deterioration of collagen content, proteoglycan amount, or histologic appearance [56]. Pearsall et al. reported on 16 refrigerated osteochondral allografts that underwent histologic and ultrastructural examination prior to implantation. The authors found an inverse correlation between matrix staining and time to implantation $(\mathrm{p}<0.05)$ and reported an average time from harvest to implantation of 30 days (range: 17 to 44 days) [57]. Despite the aforementioned animal and histologic/electron microscopy data, the authors are unaware of published data correlating pre-implantation histologic and electron microscopy findings with clinical outcome.

The use of deep frozen allografts for the treatment of osteoarticular defects has also been reported, with cited failure rates as high as $25 \%[2,15,58]$. Reasons cited for failure include slow incorporation, diminished chondrocyte survival, and subsequent matrix degeneration $[1,58]$. Pritzker and others noted that freezing cartilage kills chondrocytes [4,23,59-61]. Despite reports of poor chondrocyte viability at the time of frozen allograft implantation, we detected no significant difference in outcome between our refrigerated and frozen allograft patients. Moreover, when pre-operative scores were examined, the frozen allograft group had significantly worse scores at the time of implantation, indicating that these patients were actually doing better at the latest follow-up.

There are several weaknesses to the current study. The analysis included a limited number of patients. Therefore, statistical significance could not be determined for several variables. There was no follow-up magnetic resonance imaging of the study patients to assess incorporation of the implanted graft. Previous authors have hypothesized that early magnetic resonance imaging abnormalities represent non-specific post-operative change, whereas persistent abnormalities represent immune-related injury [62]. Further follow-up of the current patient cohort will include magnetic resonance imaging to evaluate graft incorporation and analyze the findings in relation to clinical outcome. The histological and electron microscopy evaluation was performed by a single pathologist. However, the evaluator has over 20 years of expertise in this area and interpreted all specimens blinded. Although several of the electron microscopy and histologic scores were low, these findings may be attributable to the need to refine the technique of electron microscopy and histologic assessment in articular cartilage. Finally, although the current study assessed chondrocyte viability, it did not evaluate chondrocyte function. Various authors have reported on the mechanical properties of articular cartilage, including an assessment of cartilage stiffness [63-65].

In conclusion, $76 \%$ of implanted frozen and refrigerated osteochondral allografts in the current study are still in place at 4 years after surgery. At the latest follow-up, frozen allografts are surviving as well as refrigerated grafts. The reason for this finding is unclear, as the majority of these grafts were used in salvage cases in older patients. Long term follow-up is needed to assess clinical outcome, while the use of magnetic resonance imaging may be beneficial to evaluate graft incorporation and articular cartilage integrity.

\section{References}

[1] J. E. Browne and T. P. Branch, "Surgical Alternative for Treatment of Articular Cartilage Lesions," Journal of the American Academy of Orthopaedic Surgeons, Vol. 8, No. 3, 2000, pp. 180-189.

[2] F. F. Parrish, "Allograft Replacement of All or Part of the End of a Long Bone Following Excision of a Tumor," The Journal of Bone and Joint Surgery, Vol. 55, No. 1, 1973, pp. 1-22.

[3] A. W. Pearsall, J. A. Tucker, R. B. Hester and R. J. Heitman, "Osteochondral Transplantation of the Knee: An Assessment of Graft Viability, American Orthopaedic Society for Sports Medicine Specialty Day," American Academy of Orthopaedic Surgeons, Dallas, 2002.

[4] K. P. H. Pritzker, A. E. Gross, F. Langer, S. C. Luk and J. B. Houpt, "Articular Cartilage Transplantation," Human Pathology, Vol. 8, No. 6, 1977, pp. 635-651. doi:10.1016/S0046-8177(77)80093-2

[5] T. Furakawa, D. R. Eyre, S. Koide and M. J. Glimcher, "Biochemical Studies on Repair Cartilage Resurfacing Experimental Defects in the Rabbit Knee," The Journal of Bone and Joint Surgery, Vol. 62, No. 1, 1980, pp. 7989.

[6] W. W. Curl, J. Krome, E. S. Gordon, J. Rushing, B. P. Smith and G. G. Poehling, "Cartilage Injuries: A Review of 31,516 Knee Arthroscopies," Arthroscopy, Vol. 13, No. 4, 1997, pp. 456-460. doi:10.1016/S0749-8063(97)90124-9

[7] R. W. Jackson, “Arthroscopic Treatment of Degenerative Arthritis,” In: J. B. McGinty, Ed., Operative Arthroscopy, Raven Press, New York, 1991, pp. 319-323.

[8] L. Hangody and P. Füles, "Autologous Osteochondral Mosaicplasty for the Treatment of Full-Thickness Defects of Weight-Bearing Joints. Ten Years of Experimental and Clinical Experience," The Journal of Bone and Joint Surgery, Vol. 85-A, Supplement 2, 2003, pp. 25-32.

[9] L. L. Johnson, "Surgical Arthroscopy: Principles and Prac- 
tice," Mosby, St Louis, 1986.

[10] Y. Matsusue, T. Yamamuro and H. Hama, "Arthroscopic Multiple Osteochondral Transplantation to the Chondral Defect in the Knee Associated with Anterior Cruciate Ligament Disruption," Arthroscopy, Vol. 9, No. 3, 1993, pp. 318-321. doi:10.1016/S0749-8063(05)80428-1

[11] V. Bobić, "Arthroscopic Osteochondral Autograft Transplantation in Anterior Cruciate Ligament Reconstruction: A Preliminary Clinical Study," Knee Surgery, Sports Traumatology, Arthroscopy, Vol. 3, No. 4, 1996, pp. 262264. doi:10.1007/BF01466630

[12] H. Laprell and W. Peterson, "Autologous Osteochondral Transplantation Using the Diamond Bone-Cutting System (DBCS): 6-12 Years' Follow-Up of 35 Patients with Osteochondral Defects at the Knee Joint," Archives of Orthopaedic and Trauma Surgery, Vol. 121, No. 5, 2001, pp. 248-253. doi: 10.1007/s004020000217

[13] D. Koulalis, W. Schultz, M. Heyden and F. König, "Autologous Osteochondral Grafts in the Treatment of Cartilage Defects of the Knee Joint," Knee Surgery, Sports Traumatology, Arthroscopy, Vol. 12, No. 4, 2004, pp. 329-334. doi:10.1007/s00167-003-0392-5

[14] H. L. Ma, S. C. Hung, S. T. Wang, M. C. Chang and T. H. Chen, "Osteochondral Autografts Transfer for Post-Traumatic Osteochondral Defect of the Knee-2 to 5 Years Follow-Up," Injury, Vol. 35, No. 12, 2004, pp. 1286-1292. doi:10.1016/j.injury.2004.02.013

[15] H. J. Mankin, F. S. Fogelson, A. Z. Thrasher and F. Jaffer, "Massive Resection and Allograft Transplantation in the Treatment of Malignant Bone Tumors," The New England Journal of Medicine, Vol. 294, No. 23, 1976, pp. 1247-1255. doi:10.1056/NEJM197606032942301

[16] L. Hangody, G. Kish, Z. Kárpáti, I. Udvarhelyi, I. Szigeti and M. Bély, "Mosaicplasty for the Treatment of Articular Cartilage Defects: Application in Clinical Practice," Orthopedics, Vol. 21, No. 7, 1998, pp. 751-756.

[17] L. Hangody, L. Sükösd, I. Szigeti and Z. Kárpáti, "Arthroscopic Autogenous Osteochondral Mosaicplasty," Hungarian Journal of Traumatology and Orthopaedics, Vol. 39, 1996, pp. 49-54.

[18] L. Hangody, P. Feczkó, L. Bartha, G. Bodó and G. Kish, "Mosiacplasty for the Treatment of Articular Defects of the Knee and Ankle," Clinical Orthopedics and Related Research, Vol. 391, Supplement 1, 2001, pp. 328-336. doi:10.1097/00003086-200110001-00030

[19] T. Minas and S. Nehrer, "Current Concepts in the Treatment of Articular Cartilage Defects," Orthopedics, Vol. 20, No. 6, 1997, pp. 525-538.

[20] C. J. Campbell, "The Healing of Cartilage Defects," Clinical Orthopedics and Related Research, Vol. 64, 1969, pp. 45-63.

[21] D. W. Jackson, M. J. Scheer and T. M. Simon, "Cartilage Substitutes: Overview of Basic Science and Treatment Options," Journal of the American Academy of Orthopaedic Surgeons, Vol. 9, No. 1, 2001, pp. 37-52.

[22] J. C. Garrett, "Fresh Osteochondral Allografts for Treatment of Articular Defects in Osteochondritis Dissecans of the Lateral Femoral Condyle in Adults," Clinical Orthopedics and Related Research, Vol. 303, 1994, pp. 33-37.

[23] T. Gibson, "The Transplantation of Cartilage," Journal of Clinical Pathology, Vol. 20, 1967, p. 513.

[24] J. P. Fulkerson, "Anteromedialization of the Tibial Tuberosity for Patellofemoral Malalignment," Clinical Orthopedics and Related Research, Vol. 177, 1983, pp. 176181.

[25] U. Horas, D. Pelinkovic, G. Herr, T. Aigner and R. Schnettler, "Autologous Chondrocyte Implantation and Osteochondral Cylinder Transplantation in Cartilage Repair of the Knee Joint. A Prospective, Comparative Trial," The Journal of Bone and Joint Surgery. American Volume, Vol. 85-A, No. 2, 2003, pp. 185-192.

[26] G. N. Homminga, S. K. Bulstra, P. S. M. Bouwmeester and A. J. van der Linden, "Perichondral Grafting for Cartilage Lesions of the Knee," The Journal of Bone and Joint Surgery. British Volume, Vol. 72, No. 6, 1990, pp. 1003-1007.

[27] R. Lorentzon, H. Alfredson and C. Hildingsson, "Treatment of Deep Cartilage Defects of the Patella with Periosteal Transplantation," Knee Surgery, Sports Traumatology, Arthroscopy, Vol. 6, No. 4, 1998, pp. 202-208. doi:10.1007/s001670050100

[28] F. R. Noyes, R. W. Bassett, E. S. Grood and D. L. Butler, "Arthroscopy in Acute Traumatic Hemarthrosis of the Knee: Incidence of Anterior Cruciate Tears and Other Injuries," The Journal of Bone and Joint Surgery. American Volume, Vol. 62, No. 5, 1980, pp. 687-695.

[29] S. K. Williams, D. Amiel, S. T. Ball, R. T. Allen, V. W. Wong, A. C. Chen, R. L. Sah and W. D. Bugbee, "Prolonged Storage Effects on the Articular Cartilage of Fresh Human Osteochondral Allografts," The Journal of Bone and Joint Surgery. American Volume, Vol. 85-A, No. 11, 2003, pp. 2111-2120.

[30] W. D. Bugbee and B. Khadivi, "Fresh Osteochondral Allografting in the Treatment of Osteonecrosis of the Knee, Paper No. 108," American Academy of Orthopaedic Surgeons, 71st Annual Meeting, San Francisco, March 2004.

[31] A. E. Gross, F. Langer, J. Houpt, K. Pritzker and G. Friedlaender, "Allotransplantation of Partial Joints in the Treatment of Osteoarthritis of the Knee," Transplant Proceedings, Vol. 8, Supplement 1, 1976, pp. 129-132.

[32] C. H. Herndon and S. W. Chase, "The Fate of Massive Autogenous and Homogenous Bone Grafts Including Articular Surfaces," Surgery, Gynecology \& Obstetrics, Vol. 98, No. 3, 1954, pp. 273-290.

[33] F. Langer, A. E. Gross, M. West and E. P. Urovitz, "The Immunogenicity of Allograft Knee Joint Transplants," Clinical Orthopedics and Related Research, Vol. 132, 1978, pp. 155-162.

[34] H. J. Mankin, S. Doppelt and W. Tomford, "Clinical Experience with Allograft Implantation. The First Ten Years," Clinical Orthopedics and Related Research, Vol. 174, 1983, pp. 69-86.

[35] D. W. Jackson, J. Halbrecht, C. Proctor, et al. "Assess- 
ment of Donor Cell and Matrix Survival in Fresh Articular Cartilage Allografts in a Goat Model," Journal of Orthopaedic Research, Vol. 14, No. 2, 1996, pp. 255-264. doi:10.1002/jor.1100140214

[36] F. R. Convery, M. H. Meyers and W. H. Akeson, "Fresh Osteochondral Allografting of the Femoral Condyle," Clinical Orthopedics and Related Research, Vol. 273, 1991, pp. 139-145.

[37] F. R. Convery, W. H. Akeson and M. H. Meyers, "The Operative Technique of Fresh Osteochondral Allografting of the Knee," Operative Techniques in Orthopaedics, Vol. 7, No. 4, 1997, pp. 340-344. doi:10.1016/S1048-6666(97)80038-9

[38] J. C. Garrett, "Osteochondral Allografts for Reconstruction of Articular Defects of the Knee," Instructional Course Lectures, Vol. 47, 1998, pp. 517-522.

[39] A. E. Gross, "Fresh Osteochondral Allografts for Post-Tra- umatic Knee Defects: Surgical Technique," Operative Techniques in Orthopedics, Vol. 7, No. 4, 1997, pp. 334-339.

[40] G. L. Bonney and M. Laurence, "Allograft Arthroplasty of the Knee," Proceedings of the Royal Society of Medicine, Vol. 62, No. 6, 1969, pp. 583-585.

[41] R. C. Locht, A. E. Gross and F. Langer, "Late Osteochondral Allograft Resurfacing for Tibial Plateau Fractures," The Journal of Bone and Joint Surgery. American Volume, Vol. 66, No. 3, 1984, pp. 328-335.

[42] M. H. Meyers and S. N. Chatterjee, "Osteochondral Transplantation," The Surgical Clinics of North America, Vol. 58, No. 2, 1978, pp. 429-434.

[43] M. H. Meyers, R. E. Jones, R. W. Bucholz, et al., "Fresh Autogenous Grafts and Osteochondral Allografts for the Treatment of Segmental Collapse in Osteonecrosis of the Hip," Clinical Orthopedics and Related Research, Vol. 174, 1983, pp. 107-112.

[44] K. Pap and S. Krompecher, "Arthroplasty of the Knee: Experimental and Clinical Experiences," The Journal of Bone and Joint Surgery. American Volume, Vol. 43, No. 4, 1961, pp. 523-537.

[45] B. C. Emmerson, S. Görtz, A. A. Jamali, C. Chung, D. Amiel and W. D. Bugbee, "Fresh Osteochondral Allografting in the Treatment of Osteochondritis Dissecans of the Femoral Condyle," The American Journal of Sports Medicine, Vol. 35, No. 6, 2007, pp. 907-914. doi: $10.1177 / 0363546507299932$

[46] P. C. McCulloch, R. W. Kang, M. H. Sobhy, J. K. Hayden and B. J. Cole, "Prospective Evaluation of Prolonged Fresh Osteochondral Allograft Transplantation of the Femoral Condyle: Minimum 2-Year Follow-Up," The American Journal of Sports Medicine, Vol. 35, No. 3, 2007, pp. 411-420. doi:10.1177/0363546506295178

[47] R. J. Williams, A. S. Ranawat, H. G. Potter, T. Carter and R. F. Warren, "Fresh Stored Allografts for the Treatment of Osteochondral Defects of the Knee," The Journal of Bone and Joint Surgery. American Volume, Vol. 89, No. 4, 2007, pp. 718-726. doi:10.2106/JBJS.F.00625

[48] N. Bellamy and W. W. Buchanan, "Outcome Measure- ment in Osteoarthritis Clinical Trials: The Case for Standardisation," Clinical Rheumatology, Vol. 3, No. 3, 1984, pp. 293-303. doi:10.1007/BF02032334

[49] A. Pace, N. Orpen, H. Doll and E. J. Crawfurd, "Outcome Scoring System Evaluation of Knee Osteoarthritis in $\mathrm{Pa}$ tients Awaiting TKA," The Journal of Knee Surgery, Vol. 19, No. 2, 2006, pp. 85-88.

[50] M. A. Ritter, A. E. Thong, K. E. Davis, M. E. Berend, J. B. Meding and P. M. Faris, "Long-Term Deterioration of Joint Evaluation Scores," The Journal of Bone and Joint Surgery. British Volume, Vol. 86, No. 3, 2004, pp. 438442. doi:10.1302/0301-620X.86B3.14243

[51] N. Bellamy, W. W. Buchanan, C. H. Goldsmith, J. Campbell and L. W. Stitt, "Validation Study of WOMAC: A Health Status Instrument for Measuring Clinically Important Patient Relevant Outcomes to Antirheumatic Drug Therapy in Patients with Osteoarthritis of the Hip or Knee," Journal of Rheumatology, Vol. 15, No. 12, 1988, pp. 1833-1840.

[52] N. Bellamy, W. F. Kean, W. W. Buchanan, E. Gerecz-Simon and J. Campbell, "Double Blind Randomized Controlled Trial of Sodium Meclofenamate (Meclomen) and Diclofenac Sodium (Voltaren): Post Validation Reapplication of the WOMAC Osteoarthritis Index," Journal of Rheumatology, Vol. 19, No. 1, 1992, pp. 153- 159.

[53] D. Amiel, F. L. Harwood, J. A. Hoover, et al., "A Histological and Biomechanical Assessment of the Cartilage Matrix Obtained from in Vitro Storage of Osteochondral Allografts," Connective Tissue Research, Vol. 23, No. 1, 1989, pp. 89-99. doi:10.3109/03008208909103906

[54] M. K. Kwan, J. S. Wayne, et al., "Histological and Biomechanical Assessment of Articular Cartilage from Stored Osteochondral Shell Allografts," Journal of Orthopaedic Research, Vol. 7, No. 5, 1989, pp. 637-644. doi:10.1002/jor.1100070503

[55] K. M. Oates, A. C. Chen, E. P. Young, et al., "Effects of Tissue Culture Storage on the in Vivo Survival of Canine Osteochondral Allografts," Journal of Orthopaedic Research, Vol. 13, No. 4, 1995, pp. 526-569. doi:10.1002/jor.1100130411

[56] J. S. Wayne, D. Amiel, M. K. Kwan, et al., "Long-Term Storage Effects on Canine Osteochrondral Allografts," ACTA Orthopaedica Scandinavian, Vol. 61, No. 6, 1990, pp. 539-545. doi:10.3109/17453679008993578

[57] A. W. Pearsall, J. A. Tucker, R. B. Hester and R. J. Heitman, "Chondrocyte Viability in Refrigerated Osteochondral Allografts Used for Transplantation within the Knee," The American Journal of Sports Medicine, Vol. 32, No. 1, 2004, pp. 125-131. doi: $10.1177 / 0095399703258614$

[58] V. M. Goldberg and S. Stevenson, "Natural History of Autografts and Allografts," Clinical Orthopedics and Related Research, Vol. 225, 1987, pp. 7-16.

[59] T. Gibson, "Viability of Cartilage after Freezing," Proceedings of the Royal Society B: Biological Sciences, Vol. 147, No. 929, 1957, pp. 528-529.

[60] W. H. Simon and W. T. Green, "Experimental Production 
of Cartilage Necrosis by Cold Injury: Failure to Cause Degenerative Joint Disease," The American Journal of Pathology, Vol. 64, No. 1, 1971, pp. 145-154.

[61] W. H. Simon, S. Richardson, W. Herman, et al., "Long-Term Effects of Chondrocyte Death on Rabbit Articular Cartilage in Vivo," Journal of the American Academy of Orthopaedic Surgeons, Vol. 58, No. 4, 1976, pp. 517526.

[62] C. B. Sirlin, J. Brossmann, R. D. Boutin, M. N. Pathria, F. R. Convery, W. Bugbee, R. Deutsch, L. K. Lebeck and D. Resnick, "Shell Osteochondral Allografts of the Knee: Comparison of MR Imaging Findings and Immunologic Responses," Radiology, Vol. 219, No. 1, 2001, pp. 35-43.

[63] J. H. Dashefsky, "Arthroscopic Measurement of Chon- dromalacia of Patella Cartilage Using a Microminiature Pressure Transducer," Arthroscopy, Vol. 3, No. 2, 1987, pp. 80- 85. doi:10.1016/S0749-8063(87)80021-X

[64] G. E. Kempson, H. Muir, S. A. Swanson and M. A. Freeman, "Correlations between Stiffness and the Chemical Constituents of Cartilage on the Human Femoral Head," Biochimica et Biophysica Acta, Vol. 215, No. 1, 1970, pp. 70-77.

[65] T. Lyyra, J. Jurvelin, P. Pitkänen, U. Väätäinen and I. Kviranta, "Indentation Instrument for the Measurement of Cartilage Stiffness under Arthroscopic Control," Medical Engineering \& Physics, Vol. 17, No. 5, 1995, pp. 395399. doi:10.1016/1350-4533(95)97322-G 